\title{
Review of Locking Alleviation Methods for Continuum-based ANCF Elements
}

Leonid Obrezkov ( $\sim$ leonid.obrezkov@lut.fi )

LUT-yliopisto: LUT University https://orcid.org/0000-0001-5271-5073

Aki Mikkola

LUT-yliopisto: LUT University

Marko Matikainen

LUT-yliopisto: LUT University

\section{Research Article}

Keywords: Absolute nodal coordinate, Locking problems, FE analysis, Large strains, Nonlinear elasticity

Posted Date: February 3rd, 2022

DOI: https://doi.org/10.21203/rs.3.rs-1303804/v1

License: (1) This work is licensed under a Creative Commons Attribution 4.0 International License.

Read Full License 


\title{
Review of locking alleviation methods for continuum-based ANCF elements
}

\author{
Leonid P. Obrezkov • Aki Mikkola • \\ Marko K. Matikainen
}

Received: date / Accepted: date

\begin{abstract}
The absolute nodal coordinate formulation (ANCF) is a nonlinear finite element approach proposed for the large deformation dynamics analysis of beam- and plate/shell-type structures. In the ANCF approach, elastic forces can be defined using three-dimensional elasticity based continuum mechanics. This approach is often straightforward, and it makes it possible to use advanced material models in the ANCF framework. However, it has been pointed out in several studies that continuum ANCF-based elements with a full three-dimensional elasticity description can suffer from locking phenomena. In this study, a comparison between various combinations of locking alleviation techniques and their applicability to different ANCF beam variants is studied using numerical examples. Furthermore, the enhanced deformation gradient (EDG) technique, which has been proposed recently in finite element literature, is demonstrated for high-order ANCF beam elements. Based on the numerical tests, none of currently available techniques are suitable for all types of ANCF elements. The paper also shows that the efficiency and accuracy of the techniques are case dependent. For the ANCF beam element involving higher-order terms with respect to trapezoidal mode, however, the EDG-based techniques are preferable to reduce locking phenomena.
\end{abstract}

Keywords Absolute nodal coordinate - Locking problems · FE analysis · Large strains · Nonlinear elasticity

L. Obrezkov · A. Mikkola · M. Matikainen

LUT University, School of Energy Systems, Mechanical Engineering, Lappeenranta, Finland E-mail: Leonid.Obrezkov@lut.fi

A. Mikkola

E-mail: Aki.Mikkola@lut.fi

M. Matikainen

E-mail: Marko.Matikainen@lut.fi 


\section{Introduction}

The absolute nodal coordinate formulation (ANCF) is a nonlinear finite element approach proposed by Shabana in [24] for beam- and plate/shell-type structures undergoing large deformation dynamics. Today, the ANCF elements are often used within the multibody community to solve various two- and three-dimensional deformation problems [11]. The key idea of ANCF elements is to use absolute nodal positions and their gradients in the kinematic description. Similar approaches were proposed by Rhim and Lee [23] (termed the vectorial approach) for nonlinear static problems involving finite elements and, recently, in the work [4].

The gradient vectors, the slope vectors in the transverse directions, are used to define cross-sectional deformations. They make it possible to use threedimensional elasticity descriptions for the ANCF elements. However, ANCF elements based on full three-dimensional elasticity are often unable to rightfully represent the deformation caused by the coupling of axial and transverse normal strains [27]. As a result, an inaccurate response results when Poisson's ratio is not zero. This problem is often referred to as the Poison locking effect $[23,7,19]$ etc.

The theoretical example of that is given in [15] and [28] as follows. Pure bending deformation leads to the trapezoidal shape of an initially rectangular cross-section. However, some ANCF elements cannot reproduce the trapezoidal shape, which leads to additional stresses during the bending deformations. As a result, it produces an inaccurate response when Poisson's ratio differs from zero.

Additionally, as was shown in [9], ANCF-based elements can suffer from other locking phenomena such as curvature thickness locking and shear locking. The first can be attributed to the element kinematics volume description and related to elements with the linear cross-section interpolation description. That interpolation leads to the norm reduction of the vectors, which define the cross-sectional thickness, when the ending cross sections are not parallel, and the shrinking of the intermediate cross sections. Another locking problem is shear locking, which happens with a linearly varying bending moment. In this case, some higher-order elements demonstrate quadratic shear strain distribution, which is not correct. As a result, the shear strain component will be assigned an inflated energy value, leading to smaller displacement predictions. These phenomena might influence results and require separate careful studies. Therefore, they have not been considered here.

The higher-order ANCF elements proposed in [14, 13, 25] can be used to reduce locking phenomena due to the limited cross-section deformation description of the ANCF element. The idea of a higher-order ANCF is to enrich the polynomial basis with higher-order polynomials by using the components of higher-order derivatives as additional nodal coordinates to represent more deformation modes and by describing the cross-section deformation more precisely. 
Another option is to use one of the locking alleviation techniques. So far, several methods have been proposed such as the Enhanced Assumed Strain (EAS) method [26], the Enhanced Continuum Mechanics formulation (ECM) $[10,22,17]$, and the Strain Split Method (SSM) [19]. The idea of the ECM approach lies in the concept of reduced integration. In this approach, the matrix of elastic coefficients is divided into two parts so that the first part presents Poisson coupling between the normal strains and the second part only involves thickness deformation. The strain split method is similar to the ECM, also considering the constitutive model split. However, in the case of the ECM, the same strain matrix is used for full and reduced integration parts. On the contrary, in the SSM, the different strain matrices are used. However, the main limitation of the mentioned approaches is applicable to only the KirchhoffSaint-Venant material model, which significantly limits their usage. The EAS approach was first proposed by Simo and Rifai [26]. The basic idea was to enrich the element strain field to improve its non-uniform strain conditions. The main advantage of the EAS is that it can be used in all material models. However, it increases the degrees of freedom of the elements.

In the study, the usability of locking alleviation techniques for lower-order, fully-parameterized and higher-order continuum-based ANCF beam elements is demonstrated via various benchmark problems. Furthermore, the Enhanced Deformation Gradient method (EDG), recently proposed by Pfefferkorn and Betsch [20], is implemented in the ANCF framework and compared to other techniques. The EDG is similar to the EAS but in the EDG, the strain field is enriched via deformation gradients instead of via direct modification of the strain field. Therefore, it possesses the same advantages as the EAS approach. All approaches with the additional requirements imposed on them for representative purpose are collected in Table 1 below.

\begin{tabular}{|c|c|c|c|c|c|}
\hline $\begin{array}{ll}\text { Requirements } & \text { Methods }\end{array}$ & Cont & ECM & SSM & EAS & EDG \\
\hline Additional variables & - & - & - & $\checkmark$ & $\checkmark$ \\
\hline Split of coefficient matrix & - & $\checkmark$ & $\checkmark$ & - & - \\
\hline
\end{tabular}

Table 1: Methods' imposed requirements

The introduced ANCF elements and corresponding locking alleviation methods are verified against analytical or experimental results, and their convergence speeds are compared. As a result, the most suitable compositions, those that alleviate locking and demonstrate solutions closest to analytical or experimental results, should be found by testing various combinations of the locking alleviation techniques and the $\mathrm{ANCF}$ elements. 


\section{Kinematics of ANCF beam elements}

This section explains, the kinematics of the spatial ANCF beam elements. Let $\boldsymbol{r}=\boldsymbol{r}(x, y, z)$ be the position vector field at the current configuration, and $\boldsymbol{r}$ the position vector in the initial configuration being. The connection between these vectors is:

$$
\boldsymbol{r}=\overline{\boldsymbol{r}}+\boldsymbol{u}_{h}
$$

where vector $\boldsymbol{u}_{h}$ a displacement vector. The paper considers three different elements: gradient deficient 3333 [17], fully-parametrized 3243, and high-order 3343 [7]. These four numbers in the element name abcd have the following meaning: $a$ is the dimension of the element, $b$ denotes the number of nodes, $c$ is the number of vectors used in the approximations, and $d$ is the polynomial basis used to approximate all 3 dimensions[7]. Figures 1 and2, show the kinematics of the subject elements. The nodal degrees of freedom used and the polynomial basis are also summarized in Table 2. The directional derivatives are defined as $\boldsymbol{r}_{\alpha}=\frac{\partial \boldsymbol{r}}{\partial \alpha}, \alpha=\{x, y, z\}$.

Table 2: Proposed elements with their nodal degrees of freedom and the monomial basis, where the shorthand notation $\beta=\left\{1, x, y, z, x y, x z, x^{2}\right\}$ is used

\begin{tabular}{ccc}
\hline Elements & Nodal degrees of freedom & Basis \\
\hline 3333 & $\boldsymbol{r}, \boldsymbol{r}_{y}, \boldsymbol{r}_{z}$ & $\beta \cup\left\{x^{2} y, x^{2} z\right\}$ \\
3243 & $\boldsymbol{r}, \boldsymbol{r}_{x}, \boldsymbol{r}_{y}, \boldsymbol{r}_{z}$ & $\beta \cup\left\{x^{3}\right\}$ \\
3343 & $\boldsymbol{r}, \boldsymbol{r}_{y}, \boldsymbol{r}_{z}, \boldsymbol{r}_{y z}$ & $\beta \cup\left\{x^{2} y, x^{2} y, y z, x^{2} y z\right\}$ \\
\hline
\end{tabular}

The elements are isoparametric, therefore, they can be described with the local bi-normalized coordinate system $\boldsymbol{\xi}=\{\xi, \eta, \zeta\}$ presented in Figure 1, with the ranges for the local coordinates being $[-1,1]$.

As an example of the three-node gradient deficient beam 3333, the vector $\boldsymbol{q}$ of nodal degrees of freedom can be written as:

$$
\boldsymbol{q}^{[3333]}=\left[\boldsymbol{r}^{(1)^{T}} \boldsymbol{r}_{\eta}^{(1)^{T}} \boldsymbol{r}_{\zeta}^{(1)^{T}} \boldsymbol{r}^{(2)^{T}} \boldsymbol{r}_{\eta}^{(2)^{T}} \boldsymbol{r}_{\zeta}^{(2)^{T}} \boldsymbol{r}^{(3)^{T}} \boldsymbol{r}_{\eta}^{(3)^{T}} \boldsymbol{r}_{\zeta}^{(3)^{T}}\right]^{T}
$$

The shape function matrix $\mathbf{N}_{m}$ for this element takes the form:

$$
\mathbf{N}_{m}(\xi, \eta, \zeta)=\left[\begin{array}{lllll}
N_{1} \mathbf{I} & N_{2} \mathbf{I} & N_{3} \mathbf{I} & \ldots & N_{9} \mathbf{I}
\end{array}\right]
$$

where $\mathbf{I}$ is a $3 \times 3$ identity matrix and 


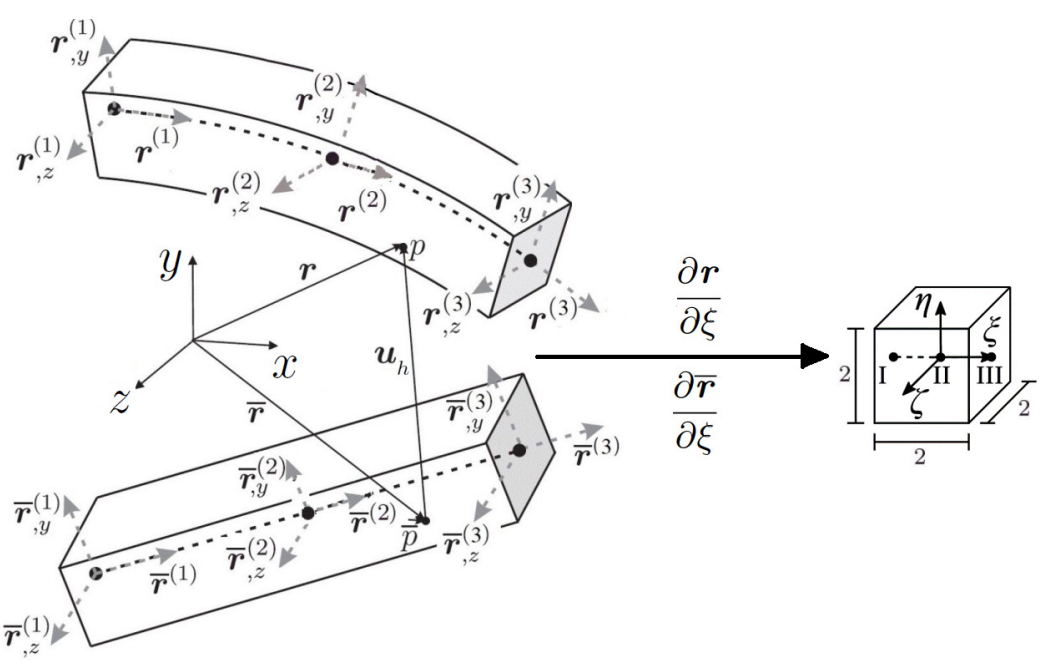

Fig. 1: Beam element with the position vector $r$ of an arbitrary particle $p$ in the current and reference configurations

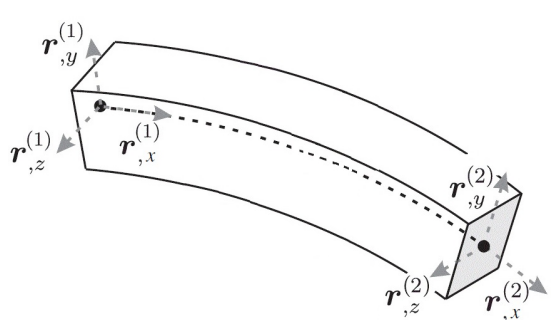

(a) fully-parametrized ANCF element 3243

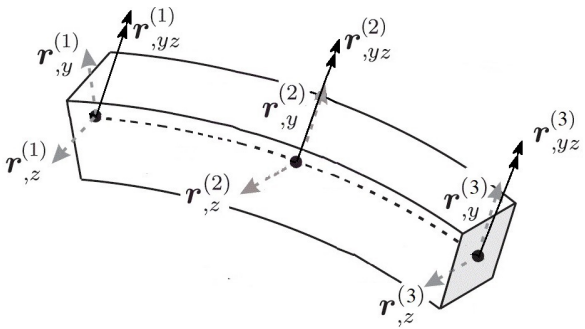

(b) high-order ANCF element 3343

Fig. 2: Illustration of the elements occurring in this study, including their nodal degrees of freedom

$$
\begin{aligned}
& N_{1}=\frac{1}{2} \xi(\xi-1) N_{2}=\frac{1}{4} l_{y} \xi \eta(\xi-1) \quad N_{3}=\frac{1}{4} l_{z} \xi \zeta(\xi-1) \\
& N_{4}=1-\xi^{2} \quad N_{5}=\frac{1}{2} l_{y} \eta\left(1-\xi^{2}\right) N_{6}=\frac{1}{2} l_{z} \zeta\left(1-\xi^{2}\right) \\
& N_{7}=\frac{1}{2} \xi(\xi+1) N_{8}=\frac{1}{4} l_{y} \xi \eta(\xi+1) N_{9}=\frac{1}{4} l_{z} \xi \zeta(\xi+1),
\end{aligned}
$$

where $l_{x}, l_{y}$, and $l_{z}$ are the physical dimensions of the element. Therefore, the finite elements can express the body motion in the form:

$$
\boldsymbol{r}(\xi, \eta, \zeta)=\overline{\boldsymbol{r}}(\xi, \eta, \zeta)+\boldsymbol{u}_{h}=\mathbf{N}_{m}(\xi, \eta, \zeta) \boldsymbol{q},
$$




\section{Equations of motion}

In this section, the equations of motion for the used elements are derived based on the principle of virtual work.

$$
\delta W_{\text {ext }}+\delta W_{\text {elast }}+\delta W_{\text {inert }}=0,
$$

where $W_{\text {ext }}$ is the virtual work by external forces, $W_{\text {elast }}$ is the virtual work by elastic forces, and $W_{\text {inert }}$ is the virtual work by inertial forces. The variation of virtual work by inertial forces with respect to the nodal coordinates can be written as follows.

$$
\delta W_{\text {inert }}=-\ddot{\boldsymbol{q}}^{T} \int_{V} \rho \mathbf{N}_{m}^{T} \mathbf{N}_{m} d V \cdot \delta \boldsymbol{q},
$$

where $\rho$ is the mass density, and $V$ is the volume of the element in the reference configuration. The integral expression is usually called the mass matrix, which has a constant meaning following from the Eq.(2).

$$
M=\int_{V} \rho \mathbf{N}_{m}^{T} \mathbf{N}_{m} d V
$$

The variation of external forces takes the following form

$$
\delta W_{e x t}=\int_{V} \boldsymbol{b}^{T} \delta \boldsymbol{r} d V=\int_{V} \boldsymbol{b}^{T} \mathbf{N}_{m} d V \cdot \delta \boldsymbol{q},
$$

where vector $\boldsymbol{b}$ is the vector of body forces.

\section{Virtual work of elastic forces}

The following paragraphs present, in detail, the elastic forces for each alleviation method.

\subsection{Standard continuum-based method}

Going forward, the general continuum mechanics based approach with full three-dimensional elasticitywill be referred to "Cont". This description suffers from Poisson locking. Modifications made to strain energy to avoid locking will be presented in the following subsections. The variation of $W_{\text {elast }}$ with respect to the nodal coordinates is

$$
\delta W_{\text {elast }}^{\text {Cont }}=\int_{V} \mathbf{S}: \delta \mathbf{E} d V=\int_{V} \mathbf{S}: \frac{\partial \mathbf{E}}{\partial \boldsymbol{q}} d V \cdot \delta \boldsymbol{q} .
$$

where $\mathbf{S}$ is the second Piola-Kirchhoff stress, and $\mathbf{E}$ is the Green-Lagrange strain, which is given as

$$
\mathbf{E}=\frac{1}{2}\left(\mathbf{F}^{T} \cdot \mathbf{F}-\mathbf{I}\right)
$$


From (3) the deformation gradient $\mathbf{F}$ is

$$
\mathbf{F}=\frac{\partial \boldsymbol{r}}{\partial \boldsymbol{\xi}}\left(\frac{\partial \overline{\boldsymbol{r}}}{\partial \boldsymbol{\xi}}\right)^{-1}=\frac{\partial \boldsymbol{r}}{\partial \boldsymbol{\xi}} J^{-1}
$$

where $J$ is the Jacobian matrix providing the transformation between the physical and local coordinate systems. The expression for the second PiolaKirchhoff stress tensor is of the form

$$
\mathbf{S}=\lambda \mathbf{I} \operatorname{tr}(\mathbf{E})+2 G \mathbf{E},
$$

where $\lambda$ and $G$ are Lame elastic coefficients, which relate to Young's modulus $E$ and Poison's coefficient $\nu$ as

$$
\lambda=\frac{E \nu}{(1+\nu)(1-2 \nu)}, G=\frac{E}{2(1+\nu)} .
$$

The expression (8) can also be represented via Voigt notation, where stresses and strains have the following vector forms.

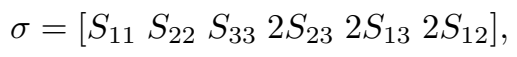

$$
\begin{aligned}
& \epsilon=\left[\begin{array}{llllll}
E_{11} & E_{22} & E_{33} & 2 E_{23} & 2 E_{13} & 2 E_{12}
\end{array}\right] .
\end{aligned}
$$

The stress-strain relation (11) can be rewritten as

$$
\sigma=\mathbf{D} \epsilon,
$$

where the elasticity matrix $\mathbf{D}$ has defined as

$$
\mathbf{D}=\left[\begin{array}{cccccc}
\lambda+2 G & \lambda & \lambda & 0 & 0 & 0 \\
\lambda & \lambda+2 G & \lambda & 0 & 0 & 0 \\
\lambda & \lambda & \lambda+2 G & 0 & 0 & 0 \\
0 & 0 & 0 & G & 0 & 0 \\
0 & 0 & 0 & 0 & G k_{s_{2}} & 0 \\
0 & 0 & 0 & 0 & 0 & G k_{s_{3}}
\end{array}\right]
$$

with shear correction factors $k_{s_{2}}, k_{s_{3}}$. Then the expression (8) has the form

$$
\delta W_{\text {elast }}^{\text {Cont }}=\frac{1}{2}\left(\int_{V} \epsilon^{T} \mathbf{D} \epsilon d V\right) \cdot \delta \boldsymbol{q} .
$$

Recalling that the element is isoparametric,

$$
\delta W_{\text {elast }}^{\text {Cont }}=\frac{1}{2}\left(\int_{-1}^{1} \int_{-1}^{1} \int_{-1}^{1} \epsilon^{T} \mathbf{D} \epsilon \operatorname{det}(J) d \xi d \eta d \zeta\right) \cdot \delta \boldsymbol{q} .
$$

An advantage of the expression (15) is that integration can be performed using the Gaussian integration, furthermore, this procedure can be applied directly and independently for the three integrations. 
4.2 Enhanced Continuum Method - ECM

The elements based on the method presented in Subsection 4.1 suffer from Poisson locking, except for some high-order ones, where the rich polynomial basis alleviates the effect naturally [7]. The ECM offers locking-free solutions based on splitting of the elasticity matrix $\mathbf{D}$. This approach was first suggested for ANCF element in [10].

$$
\mathbf{D}=\mathbf{D}^{0}+\mathbf{D}^{v}
$$

where $\mathbf{D}^{v}$ is responsible for the Poisson effect only

$$
\mathbf{D}^{v}=\left[\begin{array}{cccccc}
\frac{\lambda^{2}}{\lambda+G} & \lambda & \lambda & 0 & 0 & 0 \\
\lambda & \frac{\lambda^{2}}{\lambda+G} & \lambda & 0 & 0 & 0 \\
\lambda & \lambda & \frac{\lambda^{2}}{\lambda+G} & 0 & 0 & 0 \\
0 & 0 & 0 & 0 & 0 & 0 \\
0 & 0 & 0 & 0 & 0 & 0 \\
0 & 0 & 0 & 0 & 0 & 0
\end{array}\right]
$$

In Eq.(16), diagonal matrix $\mathbf{D}^{0}$ is as

$$
\mathbf{D}^{0}=\operatorname{diag}\left(E, E, E, G, G k_{s_{2}}, G k_{s_{3}}\right) .
$$

The strain energy variation following (16) can be also split into parts:

$$
\delta W_{\text {elast }}^{\mathrm{ECM}}=\frac{1}{2}\left(\int_{-1}^{1} \int_{-1}^{1} \int_{-1}^{1} \epsilon^{T} \mathbf{D}^{0} \epsilon \operatorname{det}(J) d \xi d \eta d \zeta+A \int_{-1}^{1} \epsilon^{T} \mathbf{D}^{v} \epsilon \frac{\partial \overline{\boldsymbol{r}}}{\partial \xi} d \xi\right) \cdot \delta \boldsymbol{q}
$$

where $A=l_{y} l_{z}$ is the cross-sectional area. This method can be considered as selective reduced integration [17] and from Eq.(19) it is clear that the Poisson effect is considered only on the beam axis $\{\eta, \zeta\}=\{0,0\}$.

\subsection{Enhanced Assumed Strain Method - EAS}

Simo and Rifai [26] proposed the enhanced assumed strain approach as a generalization of the method of incompatible modes. The key idea behind this method is improving the performance of the element with additional variables of strains via improvement of the non-uniform strain conditions. The enhanced strain field can be written in the following form

$$
\mathbf{E}=\mathbf{E}^{c o m}+\mathbf{E}^{e n h},
$$

where $\mathbf{E}^{\text {com }}$ is the compatible strain field obtained from (9) and $\mathbf{E}^{\text {enh }}$ is the enhanced strain field, which is defined per element. The enhanced strain field must be $L_{2}$ orthogonal to the discrete stress 


$$
\int_{V} \mathbf{S}: \mathbf{E}^{e n h} d V=0
$$

This eliminates the independent stress. Then, the variation of the Hu-Washizu functional type in the reference configuration, assuming that $\boldsymbol{\alpha}$ is a vector of additional enhanced strain variables gives the following system [19]:

$$
\begin{gathered}
\delta W_{\text {elast }}^{\mathrm{EAS}}=\int_{V} \mathbf{S}: \frac{\partial \mathbf{E}}{\partial \boldsymbol{q}} d V \cdot \delta \boldsymbol{q}, \\
\int_{V} \mathbf{S}: \frac{\partial \mathbf{E}}{\partial \alpha} d V \cdot \delta \alpha=0 .
\end{gathered}
$$

The solution operations are performed as follows. From the first equation of (20), the problem is solved for $\boldsymbol{q}$. Then, once $\boldsymbol{q}$ is defined, the additional enhanced strain variables $\boldsymbol{\alpha}$ are updated from the second equation of (20). The only part left here is the discretization of $\mathbf{E}^{e n h}$ field. It is assumed that

$$
\mathbf{E}^{e n h}=\mathbf{M}_{m}(\boldsymbol{\xi}) \boldsymbol{\alpha},
$$

where $\mathbf{M}_{m}$ is a shape function matrix. To alleviate the Poisson locking in this work, the linear enhanced interpolation in the element transverse directions is considered as the following.

$$
\mathbf{E}^{e n h}=\mathbf{M}_{m 2} \boldsymbol{\alpha}=\left[\begin{array}{ccc}
0 & 0 & 0 \\
0 & \eta \alpha_{1} & 0 \\
0 & 0 & \zeta \alpha_{2}
\end{array}\right] .
$$

Additionally, the second $\mathbf{M}_{m}$ matrix is assumed to be:

$$
\mathbf{M}_{m 4}=\left[\begin{array}{ccc}
0 & 0 & 0 \\
0 & \eta \alpha_{1} & \zeta \alpha_{3} \\
0 & \eta \alpha_{4} & \zeta \alpha_{2}
\end{array}\right] .
$$

In the numerical experiments, EAS methods method solutions that performed better using (22) are referred to as $E A S-2$. The solutions that performed better using (23) are referred to as $E A S-4$.

\subsection{Enhanced Deformation Gradient Method - EDG}

The enhanced deformation gradient method was previously introduced by Pfefferkorn and Betsch [20]. It is similar to the EAS. The main difference is that the enhanced field is not added to the strain field directly, but via enrichment of the deformation gradient $\mathbf{F}$ as:

$$
\mathbf{F}=\mathbf{F}^{c o m}+\mathbf{F}^{e n h}
$$

where $\mathbf{F}^{\text {com }}$ is the compatible part of the deformation gradient obtained in (10). On the other hand, the presentation of $\mathbf{F}$ might have different forms, 
various transformations are considered in [20]. In the case of the ANCF elements, however, they lead to approximately the same results. In this work, the form presented in [21] with small modification will be used as:

$$
\mathbf{F}^{e n h}=\mathbf{F}_{0}\left[\begin{array}{ccc}
0 & 0 & 0 \\
0 & \eta \alpha_{1} & 0 \\
0 & 0 & \zeta \alpha_{2}
\end{array}\right]
$$

Additionally, as it was in Section 4.3 The second version of the $\mathbf{F}^{\text {com }}$ with 4 variables is as follows:

$$
\mathbf{F}^{e n h}=\mathbf{F}_{0}\left[\begin{array}{ccc}
0 & 0 & 0 \\
0 & \eta \alpha_{1} & \zeta \alpha_{3} \\
0 & \eta \alpha_{4} & \zeta \alpha_{2}
\end{array}\right]
$$

Using the referencing system from Section 4.3, they are designated EDG-2 and $E D G-4$, respectively. Again, as in EAS approach, the system for the derivation of $\delta W_{\text {elast }}$ has here the following form:

$$
\begin{gathered}
\delta W_{\text {elast }}^{\mathrm{EDG}}=\int_{V} \mathbf{S}: \frac{\partial \mathbf{E}}{\partial \boldsymbol{q}} d V \cdot \delta \boldsymbol{q}, \\
\int_{V} \mathbf{S}: \frac{\partial \mathbf{E}}{\partial \alpha} d V \cdot \delta \alpha=0 .
\end{gathered}
$$

\subsection{Strain Split Method - SSM}

The strain split method was first presented and explained in detail in [19]. One of its main advantages is that it can cure locking without introducing the additional variables described Sections 4.3 and 4.4. The key concept of this technique is that - the axial and transverse strains of high-order terms are split via decomposition of the Green-Lagrange strain tensor (9) and the constitutive law (13). According to [29, 19], the gradient deformation tensor (10) can be separated as

$$
\mathbf{F}=\mathbf{F}^{c}+\mathbf{F}^{k}
$$

where

$$
\mathbf{F}^{c}=\left[\frac{\partial \boldsymbol{r}_{c}}{\partial \xi} \frac{\partial \boldsymbol{r}}{\partial \eta} \frac{\partial \boldsymbol{r}}{\partial \zeta}\right] J^{-1}
$$

with $\boldsymbol{r}_{c}=\left.\boldsymbol{r}\right|_{\eta, \zeta=0}$ and

$$
\mathbf{F}^{k}=\left[\frac{l_{y} \eta}{2} \frac{\partial \boldsymbol{r}}{\partial \eta \xi}+\frac{l_{z} \zeta}{2} \frac{\partial \boldsymbol{r}}{\partial \zeta \xi} 00\right] J^{-1} .
$$

Then, with help of (28) and (29) Green-Lagrange strain can be written as

$$
\mathbf{E}^{\mathrm{SSM}}=\mathbf{E}^{c}+\mathbf{E}^{k},
$$


where

$$
\begin{gathered}
\mathbf{E}^{c}=\frac{1}{2}\left(\mathbf{F}^{c T} \cdot \mathbf{F}^{c}-\mathbf{I}\right) \\
\mathbf{E}^{k}=\frac{1}{2}\left(\mathbf{F}^{c T} \cdot \mathbf{F}^{k}+\mathbf{F}^{k T} \cdot \mathbf{F}^{c}+\mathbf{F}^{k T} \cdot \mathbf{F}^{k}\right) .
\end{gathered}
$$

Using Voigt notation, the second Piola-Kirchhoff stress can be defined as

$$
\mathbf{S}^{\mathrm{SSM}}=\mathbf{D} \epsilon^{c}+\mathbf{D}^{0} \epsilon^{k}
$$

where

$$
\begin{aligned}
\epsilon^{c} & =\left[\begin{array}{llllll}
E_{11}^{c} & E_{22}^{c} & E_{33}^{c} & 2 E_{23}^{c} & 2 E_{13}^{c} & 2 E_{12}^{c}
\end{array}\right], \\
\epsilon^{k} & =\left[\begin{array}{llllll}
E_{11}^{k} & E_{22}^{k} & E_{33}^{k} & 2 E_{23}^{k} & 2 E_{13}^{k} & 2 E_{12}^{k}
\end{array}\right] .
\end{aligned}
$$

Finally, the expression for the virtual work of elastic force takes the form

$$
\delta W_{\text {elast }}^{\mathrm{SSM}}=\int_{V} \mathbf{S}^{\mathrm{SSM}}: \frac{\partial \mathbf{E}^{\mathrm{SSM}}}{\partial \boldsymbol{q}} d V \cdot \delta \boldsymbol{q},
$$

\section{Results of bending tests}

The following paragraphs analyze the performance of the techniques described in Section 4. Several often used gradient-deficient, fully-parameterized, and high-order ANCF elements are used. Their initial models, based on standard continuum approach, suffer from Poisson locking. They are referred to in the tables as "Cont".

Several benchmark problems related to the bending, presented in Figure 3 are considered.

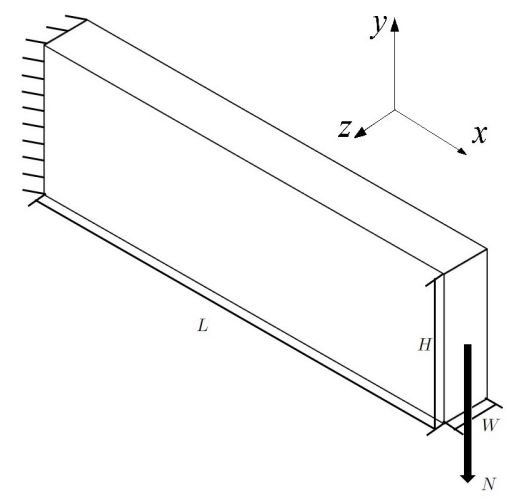

Fig. 3: Schematic illustration of the bending beam experiment 
Additionally, ANCF element 3363 is considered based on the conclusions from [7]. This element demonstrated reasonable results during deformation tests and maintained usable convergence rates.

\subsection{Small displacement cantilever problem}

The beam structure under investigation has a rectangular cross section with width $W=0.1$, height $H=0.5$, and length $L=2 . E=2.07 \times 10^{11} \frac{\mathrm{N}}{\mathrm{m}^{2}}$ and Poisson's ratio $\nu=0.3$. The small deformations case is tested first. To this end, the vertical tip load is set to $F_{y}=62.5 \times 10^{3} \mathrm{~N}$.

\begin{tabular}{|c|c|c|}
\hline Approach & Displacement & Error \% \\
\hline Cont & 0.0006056 & 25.2266 \\
ECM & 0.0008099 & 0.0004 \\
SSM & 0.0007914 & 0.0228 \\
EAS-2 & 0.0007347 & 9.2788 \\
EAS-4 & 0.0007348 & 9.2788 \\
EDG-2 & 0.0007348 & 9.2788 \\
EDG-4 & 0.0007348 & 9.2788 \\
3363 & 0.0007981 & 1.4587 \\
\hline Reference & 0.0008099 & \\
\hline
\end{tabular}

Table 3: The modifications of gradient-deficient element, ANCF 3333, for small bending test

\begin{tabular}{|c|c|c|}
\hline Approach & Displacement & Error \% \\
\hline Cont & 0.0006055 & 25.2439 \\
ECM & 0.0008097 & 0.0229 \\
SSM & 0.0007947 & 1.8733 \\
EAS-2 & 0.0007346 & 9.2974 \\
EAS-4 & 0.0007346 & 9.2974 \\
EDG-2 & 0.0007346 & 9.2974 \\
EDG-4 & 0.0007346 & 9.2974 \\
3363 & 0.0007981 & 1.4587 \\
\hline Reference & 0.0008099 & \\
\hline
\end{tabular}

Table 4: The modifications of the fully-parameterized element, ANCF 3243, for small bending test

The convergence rate against absolute error comparison for all the abovementioned elements and modifications is provided below. The absolute error is calculated in relation to the reference solutions presented in [7].

The tests of the beam subjected to small bending load revealed, that best results for all element types are obtained using the ECM approach. See Tables 


\begin{tabular}{|c|c|c|}
\hline Approach & Displacement & Error \% \\
\hline Cont & 0.0007296 & 9.9148 \\
ECM & 0.0008099 & 0.0004 \\
SSM & 0.0007914 & 2.2812 \\
EAS-2 & 0.0008013 & 1.0622 \\
EAS-4 & 0.0008018 & 1.0008 \\
EDG-2 & 0.0008013 & 1.0622 \\
EDG-4 & 0.0008023 & 0.9385 \\
3363 & 0.0007981 & 1.4587 \\
\hline Reference & 0.0008099 & \\
\hline
\end{tabular}

Table 5: The modifications of the high-order element, ANCF 3343, for small bending test

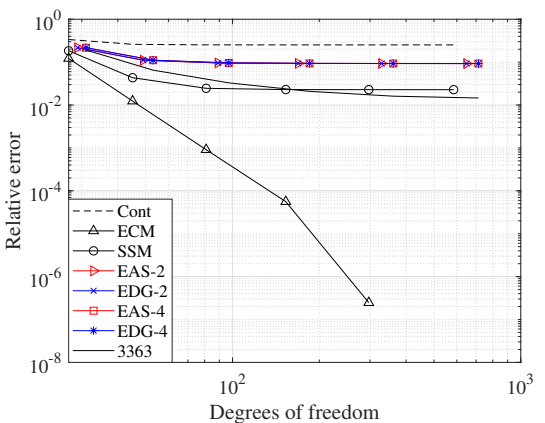

Fig. 4: Convergence rate for the gradient-deficient element, ANCF 3333, in the small bending test

3, 4 and 5 and Figures 4, 5 and 6. The ECM also provides the highest convergence rate among all approaches. However, the results presented by the other approaches for the high-order element agree within reasonable limits, with the reference solution (about 1\% error) and even outperform the high-ordered ANCF 3363 element. Additionally, including 2 or 4 degrees of freedoms into the kinematics-based locking alleviation techniques results in minimal differences for small cantilever bending problems regardless of element type. Moreover, SSM performance deteriorates as the number of vectors per node increases. 


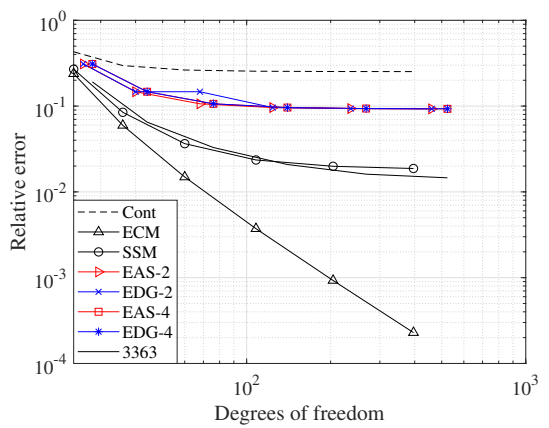

Fig. 5: Convergence rate for the fully-parameterized element, ANCF 3243, in the small bending test

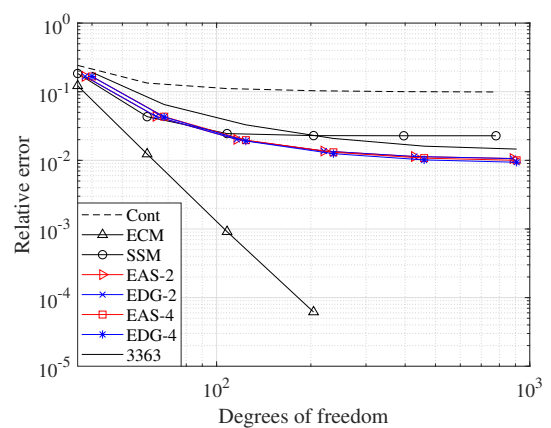

Fig. 6: Convergence rate for the high-order element, ANCF 3343, in the small bending test 
5.2 Large displacement cantilever problem

The large displacement cantilever problem is analyzed in this section. The vertical tip load is set to $F_{y}=62.5 \times 10^{6} \mathrm{~N}$. Other geometrical and physical characteristics are the same as in Section 5.1.

\begin{tabular}{|c|c|c|}
\hline Approach & Displacement & Error \% \\
\hline Cont & 0.56419 & 20.6804 \\
ECM & 0.71643 & 0.7215 \\
SSM & 0.70084 & 1.4701 \\
EAS-2 & 0.66183 & 6.9554 \\
EAS-4 & 0.66182 & 6.9554 \\
EDG-2 & 0.66221 & 6.9014 \\
EDG-4 & 0.66221 & 6.9014 \\
3363 & 0.70803 & 0.4598 \\
\hline Reference & 0.71130 & \\
\hline
\end{tabular}

Table 6: The modifications of the gradient-deficient element, ANCF 3333, for large bending test

\begin{tabular}{|c|c|c|}
\hline Approach & Displacement & Error \% \\
\hline Cont & 0.56328 & 20.8104 \\
ECM & 0.71641 & 0.7181 \\
SSM & 0.70391 & 1.0395 \\
EAS-2 & 0.66169 & 6.9741 \\
EAS-4 & 0.66169 & 6.9741 \\
EDG-2 & 0.66207 & 6.9217 \\
EDG-4 & 0.66207 & 6.9217 \\
3363 & 0.70803 & 0.4598 \\
\hline Reference & 0.71130 & \\
\hline
\end{tabular}

Table 7: The modifications of the fully-parameterized element, ANCF 3243, for large bending test

Tables 6 and 7 show that for the large bending tests, the best results for the gradient-deficient and the fully-parameterized elements are again provided by the ECM approach. Figures 7 and 8 also illustrate its high convergence rate. However, for this task, it does not perform as well as element 3363. In the case of the high-order element, from Table 8 as well as from Figure 9, the EDG4 gives the smallest error alongside the best convergence rate and performs better than that provided by the 3363 ANCF element. 


\begin{tabular}{|c|c|c|}
\hline Approach & Displacement & Error \% \\
\hline Cont & 0.65782 & 7.5187 \\
ECM & 0.71514 & 0.5403 \\
SSM & 0.70085 & 1.4687 \\
EAS-2 & 0.70869 & 0.3669 \\
EAS-4 & 0.70903 & 0.3193 \\
EDG-2 & 0.70893 & 0.3328 \\
EDG-4 & 0.70961 & 0.2375 \\
3363 & 0.70803 & 0.4598 \\
\hline Reference & 0.71130 & \\
\hline
\end{tabular}

Table 8: The modifications of the high-order element, ANCF 3343, for large bending test

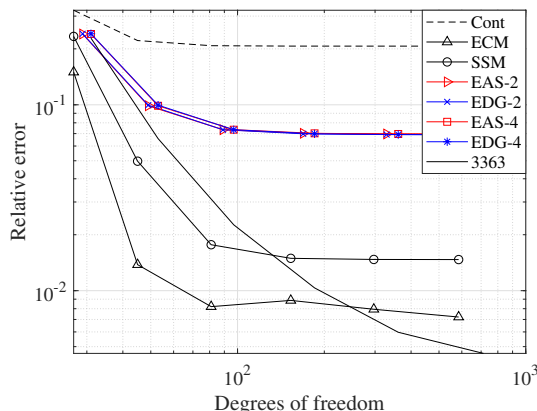

Fig. 7: Convergence rate for the gradient-deficient element, ANCF 3333, in the large bending test

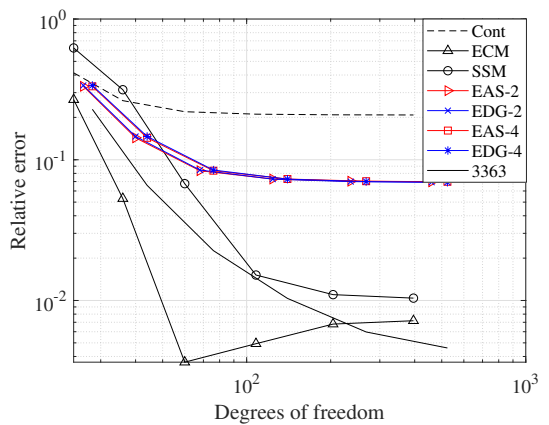

Fig. 8: Convergence rate for the fully-parameterized element, ANCF 3243, in the large bending test 


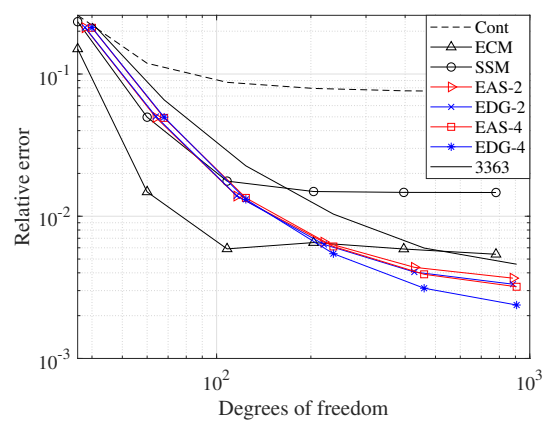

Fig. 9: Convergence rate for the high-order element, ANCF 3343, in the large bending test 


\subsection{The Princeton beam experiment}

The following paragraphs consider the Princeton beam experiment. This problem was originally conducted in [5], presented in [6], and already used within the ANCF framework by Bauchau et al. [2] and Ebel et al. [7]. In this study, beam length $L=0.508 \mathrm{~m}$. Its rectangular cross section was set to height $H=12.377 \times 10^{-3}$ and width $W=3.2024 \times 10^{-3} \mathrm{~m}$. For such a cross-section, the torsional correction factor was set to $k_{t}=0.198$ [8]. All shear correction factors were set to 1 [7]. The physical characteristics were set as follows. Young's modulus $E=71.7 \times 10^{9} \frac{\mathrm{N}}{\mathrm{m}^{2}}$ and Poisson's ratio $\nu=0.31$. In this example, several tip loads,equal to $F_{1}^{p b}=8.896$ and $F_{2}^{p b}=13.345 \mathrm{~N}$ were analyzed. An angle $\beta$ was defined as the angle between the force vector and the $\mathrm{y}$-axis, meaning that with $\beta=0^{\circ}$ the force acted in the negative $\mathrm{y}$-direction as shown by Figure 3 . The angle varied from $\beta \in\left[0^{\circ}, 90^{\circ}\right]$. The results were compared against commercial software solutions using BEAM188 and Solid185 elements.

For the calculation, 32 ANCF elements were used. The higher number of the elements leads to similar results, also following from convergence speed tests shown in Figure 4-9. The reference results for the ANSYS solutions were obtained using 128 beam elements (in the case of higher element number, see Appendix A), and $500 \times 12 \times 4$ for the model from the solid elements.

The displacements in $\mathrm{z}$ - and $\mathrm{y}$-directions of the beam under $F_{1}^{p b}$ tip load were considered.

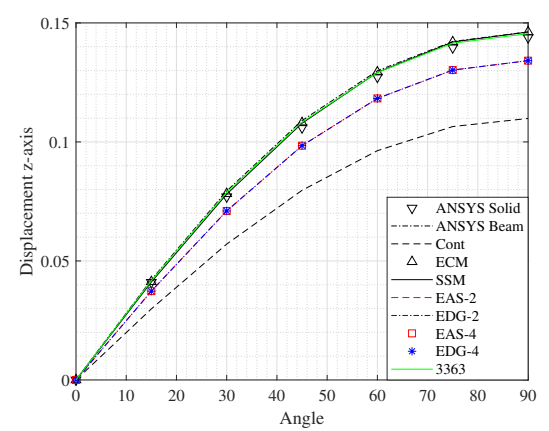

(a) in z-direction

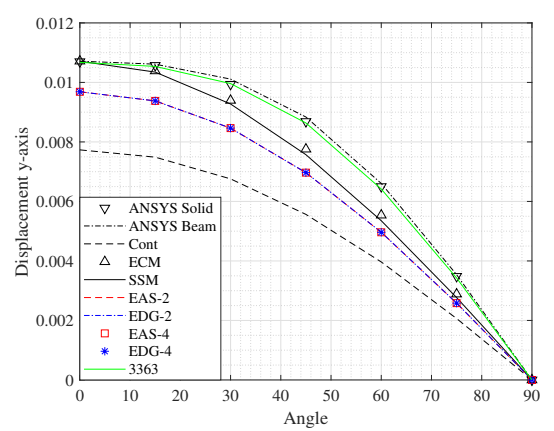

(b) in y-direction

Fig. 10: The displacements of the Princeton beam experiment of the gradientdeficient element, ANCF 3333, with modifications under $F_{1}^{p b}$

Figures 10a and 10b reveal that none of the modifications for the gradient deficient element fully passed the test. Bending along the z-axis was well represented with the ECM and SSM approaches, with results similar to the ANSYS beam, ANCF 3363, and solid element solutions. Although, the bending along the second axis was not represented well, and both these modifications resulted in poorer performance than demonstrated by the ANSYS software 
and ANCF 3363 element. Additionally, all the kinematic-based approaches, EDG and EAS, provided similar inaccurate results without differences to the number of additional DOFs.

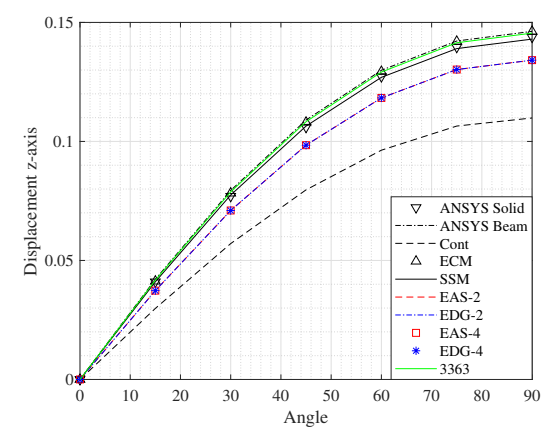

(a) in z-direction

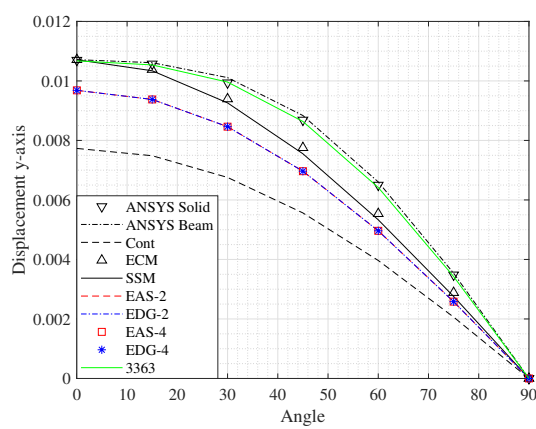

(b) in y-direction

Fig. 11: The displacements of the Princeton beam experiment of the fullyparameterized element, ANCF 3243, with modifications under $F_{1}^{p b}$

Again, as for the gradient deficient element, in the case of the fully-parameterized element, none of the modifications could correctly capture all deformations. The ECM and SSM approaches gave appropriate displacement results only along the z-axis. Furthermore, the SSM approach provided solutions similar to those provided by the solid element, and the ECM gave results similar to those obtained from the ANSYS and ANCF 3363 beam elements.

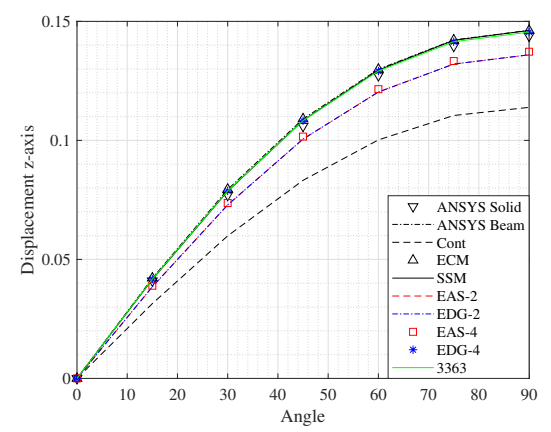

(a) in z-direction

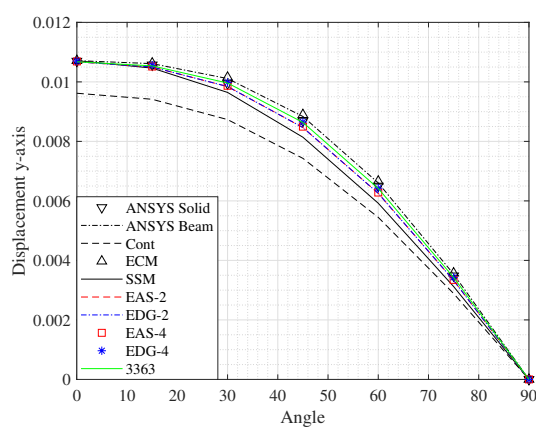

(b) in y-direction

Fig. 12: Chord-wise displacements of the Princeton beam experiment of the high-order element, ANCF 3343, with modifications under $F_{1}^{p b}$ 
For the high-order element, ANCF 3343, the best results were provided by EDG-4 and the ECM approaches, as shown by Figure 12. The ECM element demonstrated better agreement with the ANSYS beam element solution, but EDG-4 demonstrated better agreement with the ANSYS 3D solution. Additionally, in the case of the EAS approach, there were no significant differences between EAS-2 and EAS-4. The displacements given under $F_{2}^{p b}$ tip load were as follows.

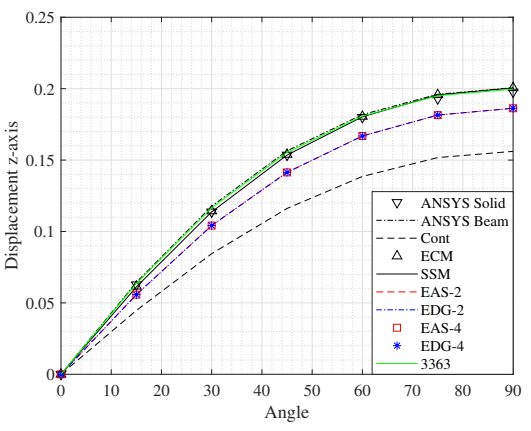

(a) in z-direction

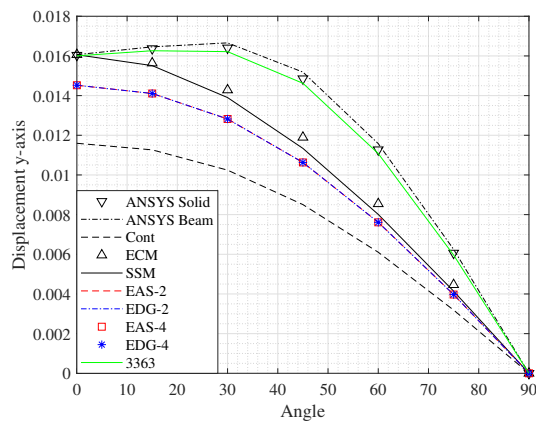

(b) in y-direction

Fig. 13: Chord-wise displacements of the Princeton beam experiment of the gradient-deficient element, $\mathrm{ANCF} 3333$, with modifications under $F_{2}^{p b}$

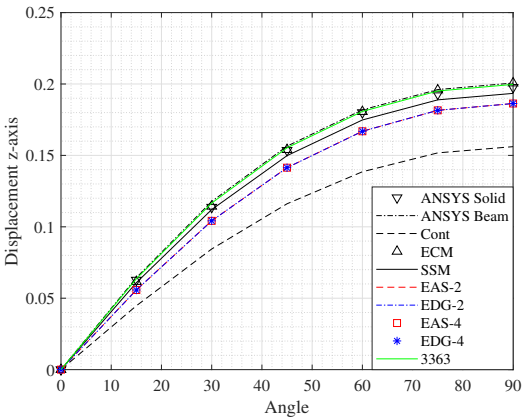

(a) in z-direction

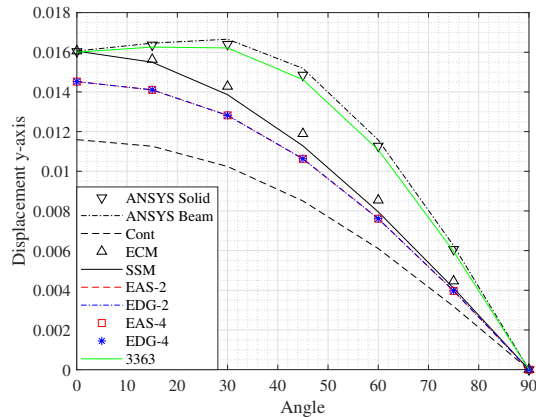

(b) in y-direction

Fig. 14: Chord-wise displacements of the Princeton beam experiment of the fully-parameterized element, ANCF 3243 , with modifications under $F_{2}^{p b}$

Similarly to the previous loading case, Figures. 13 and 14 reveal that none of the approaches cured the problems associated with bidirectional bending for low-order and fully-parameterized elements. 


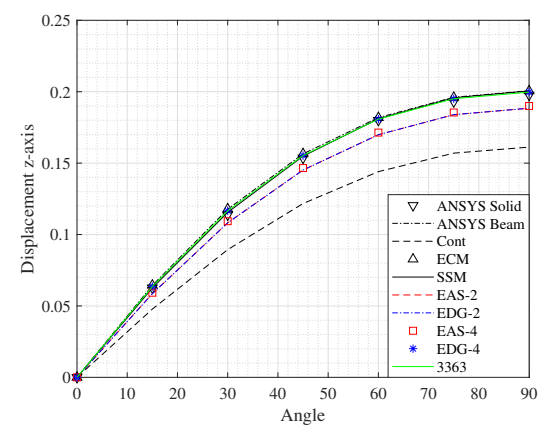

(a) in z-direction

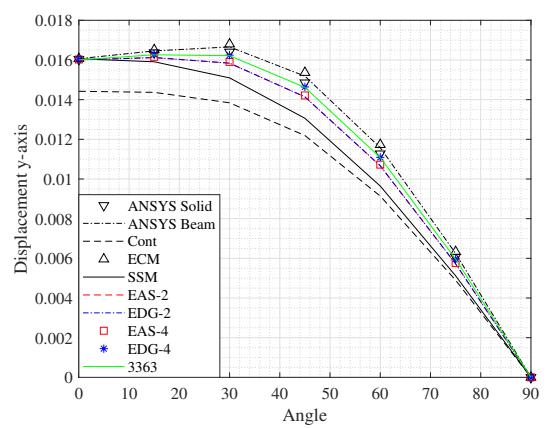

(b) in y-direction

Fig. 15: Chord-wise displacements of the Princeton beam experiment of the high-order element, ANCF 3343, with modifications under $F_{2}^{p b}$

In the case of the high-order element, ANCF 3343, the best results in the $\mathrm{z}$ - and $\mathrm{y}$-axes came from the ECM and EDG-4 approaches. The EDG-4 approach is closer to the solid element-based solution, the ECM is closer to the beam-based commercial solution.

Interestingly, regardless of the number of additional degrees of freedom, there were no significant differences in the EAS-based solutions despite element type.

\section{Eigenfrequencies of the simply supported beam}

The next numerical test was an eigenfrequencies analysis, which was used for ANCF beams in multiple works $[12,10,7,17,16,3,18]$ etc. There are multiple reasons for deciding to consider the eigenfrequency analysis. For example, Orzechowski and Shabana [18] mentioned that examining the eigenvalues might provide some explanations for locking behavior. Ebel et al. [7] stated that the eigenfrequency analysis can be used as a linearized dynamics test, because it offers the advantage of coordinate-free frequency and vibration mode comparisons. In [12], the eigenfrequency analysis was used to see if beam elements could capture the Poisson modes. The solution of the nonlinear problem depends on the set of deformation shapes assumed for the models.In this study, the example of a simply supported beam used in $[10,7,17,16,3]$ was analyzed.

A beam having a rectangular cross section with width $W=0.4$, height $H=0.4$, length $L=2$, Young's modulus $E=1 \times 10^{9} \frac{\mathrm{N}}{\mathrm{m}^{2}}$, density $\rho=7850 \frac{\mathrm{kg}}{\mathrm{m}^{3}}$, Poisson's ratio $\nu=0.3$ was analyzed. Each model was made up of 128 elements, as was done for several modifications for the gradient-deficient elements in [7]. The analytic solutions mentioned in Tables 9, 10, and 11 were based on the solutions and derivations provided in the works $[22,1]$.

Of the solutions obtained for gradient-deficient, fully-parameterized, and high-order elements, only the ECM modification was in full agreement with 


\begin{tabular}{|c|c|c|c|c|c|c|c|c|c|}
\hline Mode & 3363 & ECM & Cont & EAS-4 & EAS-2 & SSM & EDG-4 & EDG-2 & Analytic \\
\hline 1 bend & 95.41 & 95.63 & 110.27 & 100.54 & 100.54 & 97.77 & 100.54 & 100.54 & 95.63 \\
2 bend & 329.63 & 332.23 & 378.68 & 351.21 & 351.21 & 355.01 & 351.21 & 351.21 & 332.23 \\
3 bend & 625.10 & 635.70 & 717.13 & 675.39 & 675.39 & 708.48 & 675.39 & 675.39 & 635.70 \\
1 tors & 347.69 & 320.50 & 347.70 & 347.70 & 347.70 & 453.25 & 347.69 & 347.69 & 319.35 \\
2 tors & 695.39 & 641.00 & 695.39 & 695.39 & 695.39 & 906.50 & 695.39 & 695.39 & 638.70 \\
1 axis & 246.61 & 280.28 & 279.33 & 279.33 & 279.33 & 279.38 & 279.33 & 279.33 & 280.32 \\
\hline
\end{tabular}

Table 9: Eigenfrequencies of the simply supported beam for selected modes $\frac{\mathrm{rad}}{\mathrm{s}}$ of ANCF 3333 , gradient-deficient element example

\begin{tabular}{|c|c|c|c|c|c|c|c|c|c|}
\hline Mode & 3363 & ECM & Cont & EAS-4 & EAS-2 & SSM & EDG-4 & EDG-2 & Analytic \\
\hline 1 bend & 95.41 & 95.64 & 110.28 & 100.54 & 100.54 & 137.59 & 100.54 & 100.54 & 95.63 \\
2 bend & 329.63 & 332.26 & 378.71 & 351.23 & 351.23 & 494.49 & 351.23 & 351.23 & 332.23 \\
3 bend & 625.10 & 635.78 & 717.22 & 675.47 & 675.47 & 976.99 & 675.47 & 675.47 & 635.70 \\
1 tors & 347.69 & 320.51 & 347.70 & 347.70 & 347.70 & 641.01 & 347.70 & 347.70 & 319.35 \\
2 tors & 695.39 & 641.05 & 695.46 & 695.46 & 695.46 & 1282.12 & 695.46 & 695.46 & 638.70 \\
1 axis & 246.61 & 280.45 & 280.17 & 279.98 & 279.98 & 359.73 & 279.98 & 279.98 & 280.32 \\
\hline
\end{tabular}

Table 10: Eigenfrequencies of the simply supported beam for selected modes $\frac{\mathrm{rad}}{\mathrm{s}}$ of ANCF 3243 , fully-parameterized element example

\begin{tabular}{|c|c|c|c|c|c|c|c|c|c|}
\hline Mode & 3363 & ECM & Cont & EAS-4 & EAS-2 & SSM & EDG-4 & EDG-2 & Analytic \\
\hline 1 bend & 95.41 & 95.63 & 102.86 & 96.95 & 97.42 & 97.77 & 96.3 & 97.41 & 95.63 \\
2 bend & 329.63 & 332.23 & 358.16 & 340.87 & 342.22 & 355.01 & 339.03 & 342.22 & 332.23 \\
3 bend & 625.10 & 635.70 & 686.53 & 659.35 & 661.44 & 708.48 & 656.522 & 661.44 & 635.70 \\
1 tors & 347.69 & 320.50 & 347.70 & 347.69 & 347.69 & 453.25 & 347.694 & 347.69 & 319.35 \\
2 tors & 695.39 & 641.00 & 659.39 & 695.39 & 695.39 & 906.5 & 695.39 & 695.39 & 638.70 \\
1 axis & 246.61 & 280.28 & 279.33 & 279.33 & 279.33 & 279.38 & 280.18 & 279.33 & 280.32 \\
\hline
\end{tabular}

Table 11: Eigenfrequencies of the simply supported beam for selected modes $\frac{\mathrm{rad}}{\mathrm{s}}$ of ANCF 3343 , high-order element example

analytical solutions. See Tables 9, 10, and 11. that only ECM modification is in fully agreement with analytical solutions. Additionally, for the kinematicbased locking alleviation techniques EAS and EDG, the number of additional DOFs did not significantly influence the final results. 


\section{Conclusion}

Locking alleviation techniques for ANCF elements was the subject of this research. The authors considered all known techniques for continuum-based ANCF elements including the enhanced assumed strain method, the enhanced continuum mechanics approach, the strain split method, and a newly proposed enhanced deformation gradient method. These methods were applied to low-order, fully-parameterized and high-order ANCF beam elements. All possible combinations were investigated and compared. None of the modifications performed better than the others for all the presented tasks.

For small bending cases, the best results were obtained via the ECM approach for all element types. However, the results presented by other approaches for the high-order element were within reasonable limits and outperform the 3363 element. When the large bending case was considered for the low-order and fully-parameterized elements, the ECM approach again provided the best solutions. However, in the case of the high-order element, the results of the EDG-4 approach were closest to the reference with the highest convergence rate. During the Princeton beam experiments, where the bidirectional loading cases are considered, the appropriate results were demonstrated by the ECM and EDG-4 approaches for only the high-order ANCF element. Furthermore, the EDG-4 provided solutions in good agreement with the solid element, and the ECM showed good correlation with beam-based commercial software solutions. The EAS-2 and EAS-4, methods did not differ significantly regardless of considered bending loading cases and element types, which leads to the conclusion that between them, the EAS-2 is the preferable choice, because it is less computationally intensive. For eigenfrequency analysis, the best performance was ultimately demonstrated by the ECM approach.

Among the considered modifications, the locking problem can be completely cured only for the high-order element and only using the ECM and EDG-4 approaches. However, the ECM approach is limited and does not allow working with material models other than Kirchhoff-Saint-Venant. Therefore, the 3343 element with EDG-4 modification is the most universal solution. Further comparison with the element denoted 3363, and assuming as the optimal solution for various problems in [7], shows that 3343 with the EDG-4 element provides better results with the requirement of fewer DOFs. Therefore, the newly proposed approach, namely EDG-4, is a good tool to improve the performance of the high-order ANCF continuum-based beam element in the alleviation of Poisson locking in conventional load cases. Further research with practical applications to ensure the performance of the 3343 might be interesting. Additional higher-order deformation modes could be beneficial in recreating deformations caused by centrifugal forces such as rotor dynamics, etc. 


\section{Declarations}

The authors declare that they have no known competing financial interests or personal relationships that could have appeared to influence the work reported in this paper.

\section{Data declaration}

My manuscript has no associated data.

\section{References}

1. Augustyn E, Kozień MS (2015) Analytical solution of excited torsional vibrations of prismatic thin-walled beams. Journal of Theoretical and Applied Mechanics 53:991-1004

2. Bauchau OA, Han S, Mikkola A, Matikainen MK, Gruber P (2015) Experimental validation of flexible multibody dynamics beam formulations. Multibody System Dynamics 34:373-389

3. Bozorgmehri B, Hurskainen VV, Matikainen MK, Mikkola A (2019) Dynamic analysis of rotating shafts using the absolute nodal coordinate formulation. Journal of Sound and Vibration 453:214-236

4. Choi MJ, Sauer RA, Klinkel S (2021) An isogeometric finite element formulation for geometrically exact timoshenko beams with extensible directors. Computer Methods in Applied Mechanics and Engineering 385:113993

5. Dowell E, Traybar J (1975) An experimental study of the nonlinear stiffness of a rotor blade undergoing flap, lag and twist deformations. In: Technical Report 1194

6. Dowell EH, JTraybar, Hodges DH (1977) An experimental-theoretical correlation study of non-linear bending and torsion deformations of a cantilever beam. Journal of Sound Vibration 50:533-544

7. Ebel H, Matikainen MK, Hurskainen VV, Mikkola A (2017) Higher-order beam elements based on the absolut nodal coordinate formulation for three-dimensional elasticity. Nonlinear Dynamics 88:1075-1091

8. Freund J, Karakoc A (2016) Shear and torsion correction factors of timoshenko beam model for generic cross sections. Research on Engineering Structures and Materials 2:19-27

9. Garcìa-Vallejo D, Mikkola AM, Escalona JL (2007) A new locking-free shear deformable finite element based on absolute nodal coordinates. Nonlinear Dynamics 50:249-264

10. Gerstmayr J, Matikainen MK, Mikkola AM (2008) A geometrically exact beam element based on the absolute nodal coordinate formulation. Multibody System Dynamics 4:359-384 
11. Gerstmayr J, Sugiyama H, Mikkola A (2013) Review on the Absolute Nodal Coordinate Formulation for Large Deformation Analysis of Multibody Systems. Journal of Computational and Nonlinear Dynamics 8(3)

12. Maqueda LG, Shabana AA (2007) Poisson modes and general nonlinear constitutive models in the large displacement analysis of beams. Multibody System Dynamics volume 18:375-396

13. Matikainen M, Valkeapää A, Mikkola A, Schwab AL (2014) A study of moderately thick quadrilateral plate elements based on the absolute nodal coordinate formulation. Multibody System Dynamics 31:309-338

14. Matikainen MK, Dmitrochenko O, Mikkola A (2010) Beam elements with trapezoidal cross section deformation modes based on the absolute nodal coordinate formulation. AIP Conference Proceedings 1281(1):1266-1270

15. Nachbagauer K (2014) State of the Art of ANCF elements regarding geometric description, interpolation strategies, definition of elastic forces, validation and the locking phenomenon in comparison with proposed beam finite element. Archives of Computational Methods in Engineering 21:293 319

16. Nachbagauer K, Gerstmayr J (2013) Structural and continuum mechanics approaches for a 3d shear deformable ancf beam finite element: Application to buckling and nonlinear dynamic. Special Issue of the Journal of Computational and Nonlinear Dynamics 9

17. Nachbagauer K, Gruber P, Gerstmayr J (2013) A 3D shear deformable finite element based on the absolute nodal coordinate formulation. Multibody Dynamics 28:77-96

18. Orzechowski G, Shabana AA (2016) Analysis of warping deformation modes using higher order ancf beam element. Journal of Sound and Vibration 363:428-445

19. Patel M, Shabana AA (2018) Locking alleviation in the large displacement analysis of beam elements: the strain split method. Acta Mechanica 229:2923-2946

20. Pfefferkorn R, Betsch P (2019) On transformations and shape functions for enhanced assumed strain elements. International Journal for Numerical Methods in Engineering 120:231-261

21. Pfefferkorn R, Bieber S, Oesterle B, Bischoff M, Betsch P (2021) Improving efficiency and robustness of enhanced assumed strain elements for nonlinear problems. International Journal for Numerical Methods in Engineering 122(8):1911-1939

22. mand R Hertzen MM, Mikkola A, Gerstmayr J (2010) Elimination of high frequencies in the absolute nodal coordinate formulation. Proceedings of The Institution of Mechanical Engineers Part K-journal of Multi-body Dynamics 224:103-116

23. Rhim J, Lee SW (1998) A vectorial approach to computational modelling of beams undergoing finite rotations. International Journal for Numerical Methods in Engineering 41(3):527-540

24. Shabana AA (1997) Definition of the slopes and the finite element absolute nodal coordinate formulation. Multibody System Dynamics 1:339-348 
25. Shen Z, Li P, Liu C, Hu G (2014) A finite element beam model including cross-section distortion in the absolute nodal coordinate formulation. Nonlinear Dynamics 77(3):1019-1033

26. Simo JC, Rifai MS (1990) A class of mixed assumed strain methods and the method of incompatible modes. International Journal for Numerical Methods in Engineering 29:1595-1638

27. Sopanen JT, Mikkola AM (2003) Description of elastic forces in absolute nodal coordinate formulation. Nonlinear Dynamics 34

28. Sopanen JT, Mikkola AM (2003) Studies on the stiffness properties of the absolute nodal coordinate formulation for three-dimensional beams. In: International Design Engineering Technical Conferences and Computers and Information in Engineering Conference, vol Volume 5: 19th Biennial Conference on Mechanical Vibration and Noise, Parts A, B, and C, pp 209-218

29. Sugiyama H, Gerstmayr J, Shabana AA (2006) Deformation modes in the finite element absolute nodal coordinate formulation. Journal of Sound and Vibration 298:1129 - 1149

\section{A On ANSYS converge problems}

In Sec. 5.3 the authors mentioned some convergence problems within ANSYS commercial software and put the element number as 128. As a matter of fact, with increasing element number, the solutions stopped to converge to analytical solution and started significantly stepwise increasing, see in Figure 16. The authors wanted to demonstrate this convergence problem for the case of Princeton beam experiment from Section 5.3, for two rotational angles $\beta=0^{\circ}$ and $\beta=30^{\circ}$ in the case $F_{2}^{p b}$.

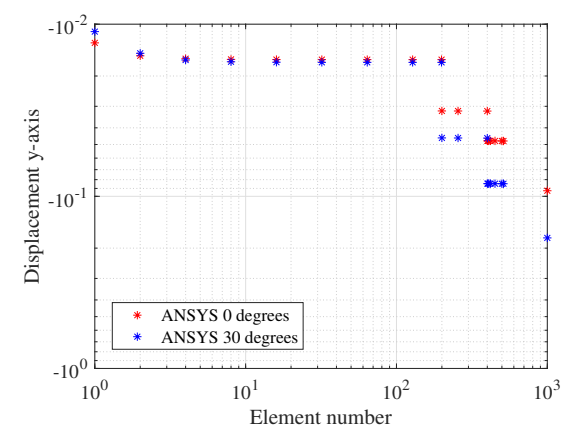

Fig. 16: The displacements from the Princeton beam experiment with dependency on the element number for $\beta=0^{\circ}$ and $\beta=30^{\circ}$

The problem was not limited by the beam presented in Section 5.3 and this feature was observed for any rod model. Such high division can be considered as unnecessary exaggeration, but for the deformation analysis of the large beams' displacements or contact beam problems, this feature should be taken into account. 